\title{
Testing some Aspects of Usability of Crowdsourced Smartphone Generated Noise Maps
}

\author{
Andrea Pődör ${ }^{1}$, András Révész ${ }^{2}$, Attila ÓcsaI $^{1}$ and Zoltán Ladomerszki ${ }^{1}$ \\ ${ }^{1}$ University of Óbuda Székesfehérvár, Hungary ·podor.andrea@amk.uni-obuda.hu \\ ${ }^{2}$ Barnsley Council, UK
}

Short paper

\begin{abstract}
In this study, the authors evaluate the usability of noise maps generated by students. There are several applications created for collecting crowdsourced noise data to generate noise maps. These applications enable scientists to use the capacity of community, although the raw data is not accessible. In this study, noise maps generated from unprofessional measurements were compared to official maps. The study confirmed that the smartphone applications tested are not capable for scientific measurement; however, tendencies suggest that calibration may lead to reasonably accurate noise level capture. The study also revealed that different mobile applications produce different outputs. This type of user generated noise measurements cannot substitute professional surveys, but provide additional data for strategic noise mapping and monitoring.
\end{abstract}

\section{Introduction}

Noise pollution is one of the main and growing environmental problems in urban areas. Noise affects everyday life, well-being, and it can even cause severe psychological problems (Silvia et al. 2003). According to the EnVIRONMENTAL Noise Directive OF THE EUROPEAN UNION 2002/49/EG (END) each agglomeration with a population over 250000 (in Hungary more than 100000 ) - should create a noise map. The directive regulates the process of the measurements and the kind of improvements that should be proposed by the local government in order to reduce the annoyance of noise pollution. According to the Hungarian Government Decree 280/2004 (X.20), all these maps should have been prepared by 2012 , and there is an order to renew them every five years from 2010 onwards. However, due to the lack of financial resources, local authorities are unable to fund the renewal of these maps. In this case, crowdsourcing may be a good solution to contribute the legislation. (PÖDÖR \& RÉVÉSZ 2014)

The concept of using humans as sensors has a growing importance in the European Union. There are several applications of such approaches in ecology, species survey, etc. The European Commission has also funded projects to determine how suitable crowdsourced data is for risk management and monitoring deforestation.

In a previous study, the authors compared the results of noise measurements using professional equipment (Brüel \& Kjaer sound level meter type 2250) and mobile phones at 150

GI_Forum - Journal for Geographic Information Science, 1-2015.

(C) Herbert Wichmann Verlag, VDE VERLAG GMBH, Berlin/Offenbach. ISBN 978-3-87907-558-4.

(C) ÖAW Verlag, Wien. ISSN 2308-1708, doi:10.1553/giscience2015s354. 
locations. The results showed that it seems to be feasible for mobile phone measured values to approximately approach the scientifically correct values, with appropriate calibration measures. (PÖDÖR \& RÉVÉSZ 2014)

Also in the field of noise mapping several attempts were made to involve citizens through developing sensor networks such as NoiseSpy (KANJO 2010). Other attempts were focused on motivating users to participate, like NoiseBattle and NoiseQuest (GARCIA MARTI et al. 2013). KAROLUS (2013) introduced a machine learning technique in his thesis, and collected noise measurements by a participatory sensing application to determine the noisiness factor of an area. A very similar approach to this is Ear-Phone noise mapping application (RAJIB et al. 2010).

All these applications use the citizens as sensors, and although the raw measurements are not available, they can also be beneficial for other researchers. None of the above mentioned research investigated the accuracy of the measurements; therefore, the recent study aims to (1) investigate the suitability of data measurement capabilities of an average smartphone, (2) compare the measurements of freely available mobile applications potentially suitable for noise mapping, and (3) compare official noise maps and interpolated noise maps generated from a smartphone survey.

\section{Methods}

The effect of noise on humans varies via the spectrum of wavelength or frequency. It is a common practice to apply a frequency dependent weighting. The most common weighting used in noise measurements is A-Weighting. According to END, the most important indicators are Lday, Levening, Lnight and Lden in preparing the strategic noise maps. The Lday, Levening, and Lnight are A-weighted long-term average sound level as defined in ISO 1996-2: 1987, determined over the indicated period of the day, where day is 12, evening is 4 , and night is 8 hours long. In a professional survey, several technical parameters are in control, which cannot be expected from the public, however, it is important that at least two minimum requirements are met during the measurements: (1) wind speed must be less than $5 \mathrm{~m} / \mathrm{s}$, and (2) it must not be raining. [284/2007.]

\subsection{Comparing the Professional Equipment and Smartphones}

The survey was carried out in 2014 in Budapest using a Voltcraft sl - 100 sound level meter, Samsung Galaxy Xcover 2, and an IPhone 4. During the survey, 143 measurments were taken. The noise level was measured by the Sound Meter PRO application on the Samsung phone, and by the DBlevel application on the IPhone.

\subsection{Comparing Different Mobile Applications and Smartphones}

The goal of the second survey was to compare the measurements of 2 mobile applications on the same type of smartphones (Sony Xperia P). There are several applications for Android equipment with different features. We chose (1) Sound Meter PRO by Mobile Essentials, and (2) Noise meter by JINASYS. The applications use the built-in microphone, and 
measure noise volume in decibels $(\mathrm{dB})$. It is important to note that built-in microphones are optimized to human voice $(300-3400 \mathrm{~Hz}, 40-60 \mathrm{~dB})$. Therefore, the hardware has a limitation, meaning the maximum values cannot exceed 80-100 dB.

The measurements were carried out at the same time and location. 1 minute continuous measurements were averaged in every 10 minutes, in 5 time intervals during the day (from morning to night: 6-7, 11-12, 14-15, 17-18 and 21-22 o'clock). The data was recorded at a distance of $7-8 \mathrm{~m}$ from the axis of the traffic line at the busiest intersection in Gyula, Southern Hungary.

\subsection{Comparing Official and Student Generated Noise Map and Measurements}

In this study, two noise measurements were carried out by students in 2013 in Székesfehérvár (51 test points), and in 2014 in Budapest (141 test points). An official noise map and a conflict map are available in both cities. Both locations were at places with blockhouses, with high number of residents and affected by heavy traffic. These maps were compared to the results of the students' measurements.

\section{$3 \quad$ Results}

Comparing the results of the scientific measurements and the measurements by smart phones, a constant deviation between various sensor equipment in $\mathrm{dB}$ can be observed (Figure 1/A).

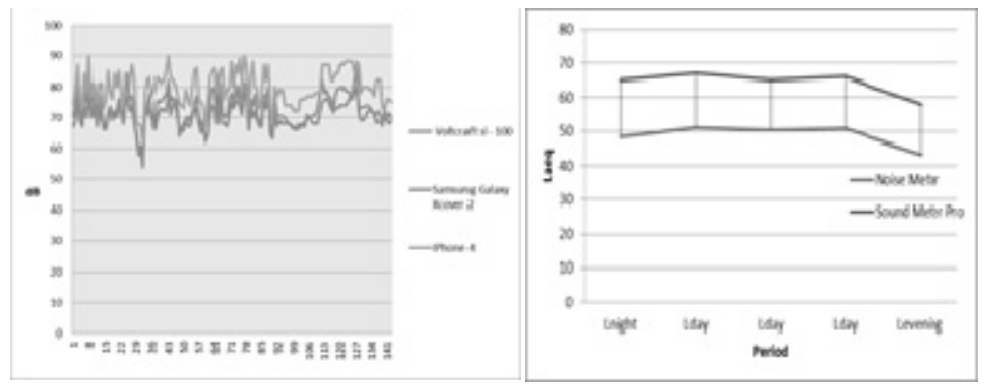

Fig. 1: A. The results of professional equipment and two smartphones.

B. The result of continuous measurements of LAeq(average sound level) of similar smart phones using different software.

There are also significant correlations between the 2 different mobile application measurements in case of LAmin ( $\mathrm{p}=8.3 \mathrm{E}-09)$ and LAeq $(\mathrm{p}=0.0097)$. The correlation coefficients of LAmin $(\mathrm{r}=0.79)$ and LAeq $(\mathrm{r}=0.43)$ suggest that there are probable positive correlations between the measurements of the 2 mobile applications. The deviation between the values was approximately $10-15 \mathrm{~dB}$. Since other circumstances were the same, it is most likely due to the lack of calibration. It seems to be feasible that with appropriate calibration, the values can approximately approach the scientifically correct values. 


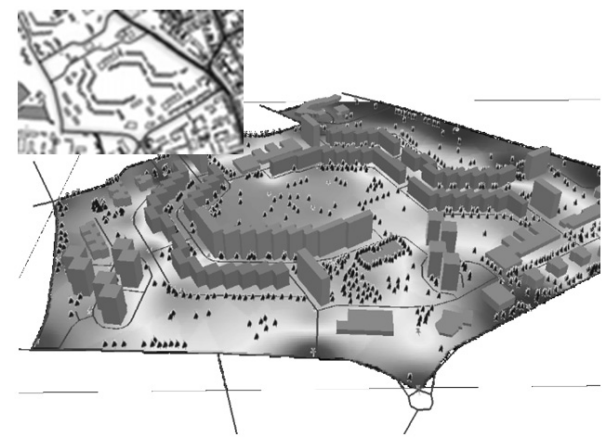

Fig. 2:

B. Student generated 3D map with the official noise map in the upper left corner

In Budapest we did not have the official data, so only visual comparisons could be made. It only shows a difference compared to the raster map in case of the highest values of over 80 $\mathrm{dB}$, measured by the student.

We also created the 3D model (Figure 2.) of the block house area with the different heights of the block houses in Székesfehérvár. The students surveyed the whole area so the model reveals that the noise level is much lower at the area surrounded by high buildings. It is not evident from the official noise map.

\section{Conclusion and Outlook}

In order to receive the same results it is beneficial to develop a customized application. It ensures that the function to calculate the noise levels is always the same, and calibration can be enforced. Further advantages of a customised application are the enforcement of following the same protocol, in order to be able to collect consequent data and take the characteristics of different equipment into consideration. Crowdsourcing can be a good option to involve citizens in measuring the noise exposure in their surroundings. This study suggests that standardizing the protocol of the survey may lead to reliable measurements, however, more investigations are required. We presumed that the characteristics of the sensors built in the same model of smartphones are the same, but this assumption needs to be checked. In a crowdsourced experiment, members of the public volunteering to survey will use different brands and models of smartphones, so further tests are necessary to compare the characteristics of the potentially different sensors.

\section{References}

ENVIRONMENTAL NOISE DIRECTIVE OF THE EUROPEAN UNION 2002/49/EG http://ec.europa.eu/environment/noise/directive.htm (accessed 02.01.2015.)

Garcia Martí, I., Rodríguez, L. E., Benedito, M., Trilles, S., Beltrán, A., Díaz, L. \& HUERTA J. (2012), Mobile application for noise pollution monitoring through gamification techniques. In ICEC, Lecture Notes in Computer Science. Springer Berlin/Heidelberg. 
KANJO E. NOISESPY (2010), A real-time mobile phone platform for urban noise monitoring and mapping. Journal of Mobile Networks and Applications, 15 (4).

KAROLUS J. (2013), Creating noise pollution maps based on user-generated noise measurements. BA Thesis, Technische Universität Darmstadt.

NOISE MAP OF SZÉKESFEHÉRVÁR http://onkormanyzat.szekesfehervar.hu/index.php?pg=page_90875 (accessed 02.01.2015.)

PŐDÖR, A. \& RÉVÉSZ, A. (2014), Noise map: professional versus crowdsourced data (Huerta, Schade, Granell (Eds), Connecting a Digital Europe through Location and Place. Proceedings of the AGILE'2014 International Conference on Geographic Information Science 2014, Castellón, June, 3-6. ISBN 978-90-816960-4-3, 2014-06)

ÓCSAI, A. (2013), Analyses noise measurments with GIS. BA Thesis, University of West Hungary, Székesfehérvár.

Rajib, K. R., Chun, T. Ch., Salil, S. K., Nirupama, B., \& Wen, H. (2010), Ear-phone: an end-to-end participatory urban noise mapping system. In ACM/IEEE IPSN.

SilviA, R., RicARDO, H. \& LUIS, C. J. (2003), Evaluation and prediction of noise pollution levels in urban areas of Cdiz (Spain). Acoustical Society of America Journal, 114, 2439. 